\title{
A synthetic electric force acting on neutral atoms
}

\author{
Y-J. Lin' ${ }^{1}$ R. L. Compton ${ }^{1}$, K. Jiménez-García ${ }^{1,2}$, W. D. Phillips ${ }^{1}$, J. V. Porto ${ }^{1}$ and I. B. Spielman ${ }^{1 \star}$
}

Electromagnetism is a simple example of a gauge theory where the underlying potentials (the vector and scalar potentials) are defined only up to a gauge choice. The vector potential generates magnetic fields through its spatial variation and electric fields through its time dependence'. Here, we report experiments in which we have produced a synthetic gauge field. The gauge field emerges only at low energy in a rubidium BoseEinstein condensate: the neutral atoms behave as charged particles do in the presence of a homogeneous effective vector potential $^{2}$. We have generated a synthetic electric field through the time dependence of an effective vector potential, a physical consequence that emerges even though the vector potential is spatially uniform.

Gauge theories play a central role in modern quantum physics. In some cases, they can be viewed as emerging as the low-energy description of a more complete theory ${ }^{3,4}$. Electromagnetism is the best known gauge theory and its gauge fields are the ordinary scalar and vector potentials. Magnetic fields arise only from spatial variations of the vector potential, whereas electric fields arise from both time variations of the vector potential and gradients of the scalar potential. These potentials are defined only to within a gauge choice, where for a charged particle the canonical momentum (the variable canonically conjugate to position) and the mechanical momentum (the mass times the velocity) are not equal. Our experiments ${ }^{2}$ have realized a particular version ${ }^{5}$ of a class of proposals ${ }^{6-11}$ to generate effective vector potentials for neutral atoms through interactions with laser light, and have created synthetic magnetic fields ${ }^{12}$ important for simulating charged condensed-matter systems with neutral atoms $s^{13,14}$. Here we demonstrate the complementary phenomenon: a synthetic electric field generated from a time-dependent effective vector potential. Additionally, we make independent measurements of both the mechanical momentum and canonical momentum, where the latter is usually not possible.

The electromagnetic vector potential $\mathbf{A}$ for a charged particle appears in the Hamiltonian $H=\left(\mathbf{p}_{\mathrm{can}}-q \mathbf{A}\right)^{2} / 2 m$, where $\mathbf{p}_{\mathrm{can}}$ is the canonical momentum, $q$ is the charge and $m$ is the mass. ( $\mathbf{p}_{\text {can }}-q \mathbf{A}=m \mathbf{v}$ is the mechanical momentum for a particle moving with velocity v.) We recently demonstrated a technique to engineer Hamiltonians of this form for ultracold atoms, and prepared a Bose-Einstein condensate (BEC) at rest with an effective vector potential $\mathbf{A}=A_{x} \hat{x}$ constant in time and space $^{2}$, corresponding to $\mathbf{E}=\mathbf{B}=0$, where $\mathbf{E}$ and $\mathbf{B}$ are the synthetic electric field and synthetic magnetic field for neutral atoms, respectively. In ref. 12, we made $\mathbf{A}$ depend on position, giving $\mathbf{B}=\nabla \times \mathbf{A} \neq 0$ but $\mathbf{E}=0$. Here we add time dependence to a spatially uniform vector potential $\mathbf{A}(t)=A(t) \hat{x}$, generating a synthetic electric field $E(t) \hat{x}=-\partial \mathbf{A} / \partial t$. The resulting force is distinct from that arising from gradients of scalar potentials $\phi(\mathbf{r})$, for example, from an external trapping potential. A revealing analogue is that of an infinite solenoid of radius
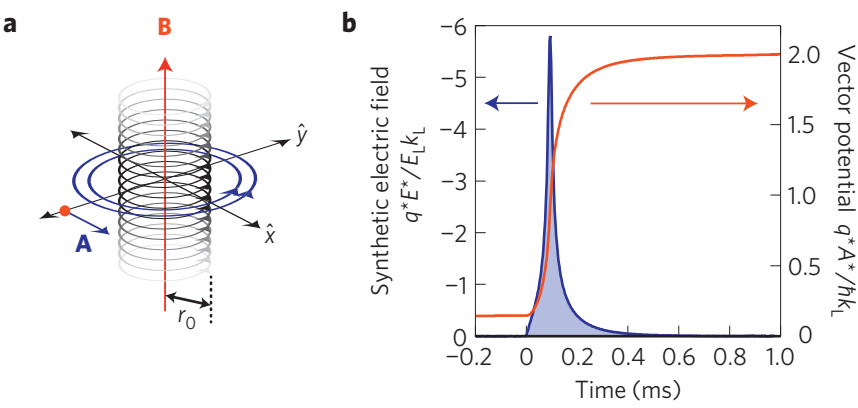

Figure 1 | Schematic diagram of the electric field generated by a time-varying vector potential. a, Emulated system, showing the electric current flowing anticlockwise in the infinite solenoid (black coil) with radius $r_{0}$ and the real magnetic field $\mathbf{B}$ only inside the solenoid. The blue lines represent the vector potential A. A charged particle (red dot) located far from the coil experiences a nearly uniform A. b. Calculated time response of the synthetic vector potential and electric field for neutral atoms in our first measurement (see Fig. 3). The calculation includes the known inductive response time of the bias field $B_{0}$, which sets the detuning, and the calibration of detuning to vector potential shown in Fig. $2 \mathrm{~d}$.

$r_{0}$ as pictured in Fig. 1a: a magnetic field $\mathbf{B}=B \hat{z}$ exists only inside the coil; however, a non-zero cylindrically symmetric vector potential $\mathbf{A}=B r_{0}{ }^{2} \hat{\phi} / 2 r$ extends outside the coil. Far from the coil $\mathbf{A}$ is nearly uniform, analogous to our uniform effective vector potential. When the current is changed in a time interval $\Delta t, \mathbf{B}$ changes with it and therefore $\mathbf{A}$ changes by $\Delta \mathbf{A}$. A charged particle on the $\hat{y}$ axis feels an electric field $-(\partial A / \partial t) \hat{x}$ during $\Delta t$, leading to $\Delta \mathbf{p}=-q \Delta A \hat{x}$, a change in the mechanical momentum even outside the solenoid.

We synthesize electromagnetic fields for neutral atoms by illuminating a ${ }^{87} \mathrm{Rb}$ BEC with two intersecting laser beams (Fig. 2a) that couple together three atomic spin states within the electronic ground state (Fig. 2b). The three new energy eigenstates, or 'dressed states', are superpositions of the uncoupled spin and linear-momentum states and have modified energy-momentum dispersion relations compared with those of uncoupled atoms. The dressed atoms act as particles with a single well-defined velocity $\mathbf{v}$, which is the population-weighted average of all three spin components.

The dispersion relation of the lowest-energy dressed state changes near its minimum, from $\mathbf{p}^{2} / 2 m$ to $\left(\mathbf{p}-\mathbf{p}_{\min }\right)^{2} / 2 m^{*}$ (Fig. 2c), where the minimum location $\mathbf{p}_{\min }$ plays the role of $q$ A. In addition, the mass $m$ is modified to an effective mass $m^{*}>m$, and both $\mathbf{p}_{\min }$ and $m^{*}$ are under experimental control (not independently). We identify $\mathbf{p}_{\min }=q^{*} \mathbf{A}^{*}$, the product of an effective charge $q^{*}$ and an effective vector potential $\mathbf{A}^{*}$ for the dressed neutral atoms. As we change $\mathbf{A}^{*}$, we induce a synthetic

\footnotetext{
${ }^{1}$ Joint Quantum Institute, National Institute of Standards and Technology, and University of Maryland, Gaithersburg, Maryland, 20899, USA,

${ }^{2}$ Departamento de Física, Centro de Investigación y EstudiosAvanzados del InstitutoPolitécnico Nacional, México D.F. 07360, México.

*e-mail: ian.spielman@nist.gov.
} 

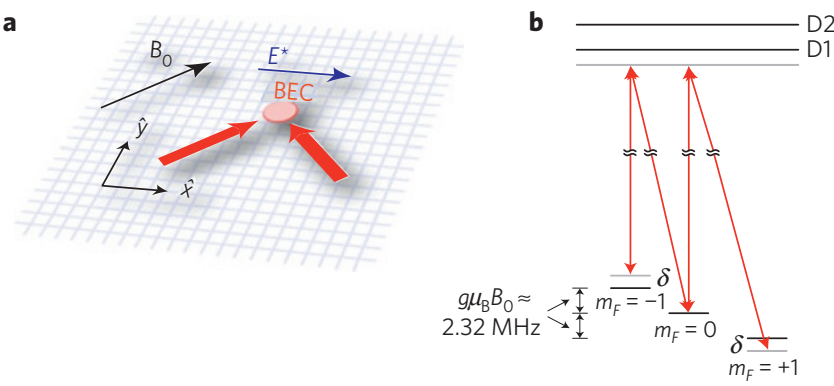

C

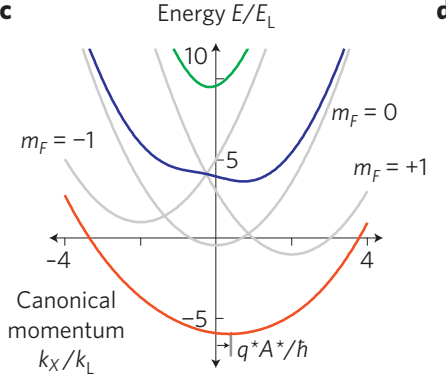

d

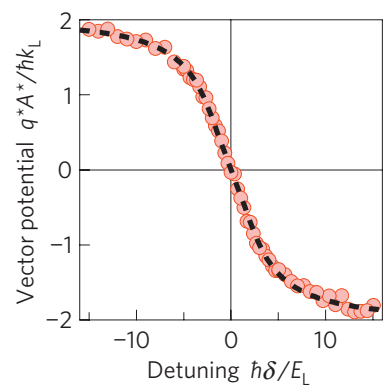

Figure 2 | Experimental setup for synthetic electric fields. a, Physical implementation indicating the two Raman laser beams incident on the BEC (red arrows) and the physical bias magnetic field $B_{0}$ (black arrow). The blue arrow indicates the direction of the synthetic electric field $E^{*}$. $\mathbf{b}$, The three $m_{F}$ levels of the $F=1$ ground-state manifold are shown as coupled by the Raman beams. c, Dressed-state eigenenergies as a function of canonical momentum for the realized coupling strength of $\hbar \Omega_{R}=10.5 E_{L}$ at a representative detuning $\hbar \delta=-1 E_{\mathrm{L}}$ (coloured curves). The grey curves show the energies of the uncoupled states, and the red curve depicts the lowest-energy dressed state in which we load the BEC. The black arrow indicates the dressed BEC's canonical momentum $p_{\text {can }}=q^{*} A^{*}$, where $A^{*}$ is the vector potential. d, Vector potentials as measured from the canonical momentum.

electric field $\mathbf{E}^{*}=-\partial \mathbf{A}^{*} / \partial t$, and the dressed BEC responds as $\mathrm{d}\left(m^{*} \mathbf{v}\right) / \mathrm{d} t=-\nabla \phi(\mathbf{r})+q^{*} \mathbf{E}^{*}$, where $\mathbf{v}$ is the velocity of the dressed atoms and $m^{*} \mathbf{v}=\mathbf{p}_{\text {can }}-q^{*} \mathbf{A}^{*}$. Here, $\Delta\left(m^{*} \mathbf{v}\right)=-q^{*}\left(\mathbf{A}_{\mathrm{f}}{ }^{*}-\mathbf{A}_{\mathrm{i}}{ }^{*}\right)$ is the momentum imparted by $q^{*} \mathbf{E}^{*}$.

We study the physical consequences of sudden temporal changes of the effective vector potential for the dressed BEC. These changes are always adiabatic such that the BEC remains in the same dressed state. We measure the resulting change of the BEC's momentum, which is in complete quantitative agreement with our calculations and constitutes the first observation of synthetic electric fields for neutral atoms.

Our system (see Fig. $2 \mathrm{a}$ ) consists of an $F=1{ }^{87} \mathrm{Rb}$ BEC with about $1.4 \times 10^{5}$ atoms initially at rest ${ }^{15,16}$; a small physical magnetic field $B_{0}$ Zeeman-shifts each of the spin states $m_{\mathrm{F}}=0, \pm 1$ by $E_{0, \pm 1}$. Here, $B_{0} \approx 3.3 \times 10^{-4} \mathrm{~T}$ and $E_{-1} \approx-E_{+1} \approx g \mu_{\mathrm{B}} B_{0} \gg\left|E_{0}\right|$. The linear and quadratic Zeeman shifts are $\hbar \omega_{\mathrm{Z}}=\left(E_{-1}-E_{+1}\right) / 2 \approx h \times 2.32 \mathrm{MHz}$ and $-\hbar \epsilon=E_{0}-\left(E_{-1}+E_{+1}\right) / 2 \approx-h \times 784 \mathrm{~Hz}$. A pair of laser beams with wavelength $\lambda=801 \mathrm{~nm}$, intersecting at $90^{\circ}$ at the BEC, couples the $m_{\mathrm{F}}$ states with strength $\Omega_{\mathrm{R}}$. These Raman lasers differ in frequency by $\Delta \omega_{\mathrm{L}} \approx \omega_{\mathrm{Z}}$ and we define the Raman detuning as $\delta=\Delta \omega_{\mathrm{L}}-\omega_{\mathrm{Z}}$. Here $\hbar \Omega_{\mathrm{R}} \approx 10 E_{\mathrm{L}}$ and $|\hbar \delta|<60 E_{\mathrm{L}}$, where $E_{\mathrm{L}}=\hbar^{2} k_{\mathrm{L}}^{2} / 2 m=h \times 3.57 \mathrm{kHz}$ and $k_{\mathrm{L}}=\sqrt{2} \pi / \lambda$ are natural units of energy and momentum.

When the atoms are rapidly moving or the Raman lasers are far from resonance $\left(k_{\mathrm{L}} v\right.$ or $\left.\delta \gg \Omega_{\mathrm{R}}\right)$, the lasers hardly affect the atoms. However, for slowly moving and nearly resonant atoms the three uncoupled states transform into three new dressed states. The spin and linear-momentum state $\left|k_{x}, m_{\mathrm{F}}=0\right\rangle$ is coupled to states $\left|k_{x}-2 k_{\mathrm{L}}, m_{\mathrm{F}}=+1\right\rangle$ and $\left|k_{x}+2 k_{\mathrm{L}}, m_{\mathrm{F}}=-1\right\rangle$, where $\hbar k_{x}$ is

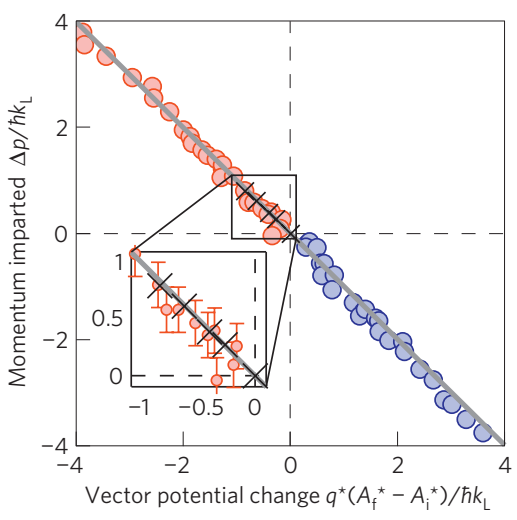

Figure 3 | Change in momentum from the synthetic electric-field kick. Three distinct sets of data were obtained by applying a synthetic electric field by changing the vector potential from $q^{*} A_{i}{ }^{*}$ (between $+2 \hbar k_{L}$ and $-2 \hbar k_{\mathrm{L}}$ ) to $q^{*} A_{f}{ }^{*}$. Circles indicate data where the external trap was removed right before the change in $A^{*}$, where $q^{*} A_{f}{ }^{*}= \pm 2 \hbar k_{L}$ ( - for red, + for blue symbols). The black crosses, more visible in the inset, show the amplitude of canonical momentum oscillations when the trapping potential was left on after the field kick. The standard deviations are also visible in the inset. The grey line is a linear fit to the data (circles) yielding slope $-0.996 \pm 0.008$, where the expected slope is -1 .

the momentum of $\left|m_{\mathrm{F}}=0\right\rangle$ along $\hat{x}$, and $2 \hbar k_{\mathrm{L}} \hat{x}$ is the momentum difference between the two Raman beams. For each $k_{x}$, the three dressed states are the energy eigenstates in the presence of Raman coupling $\hbar \Omega_{\mathrm{R}}$ (see ref. 2), with energies $E_{j}\left(k_{x}\right)$ shown in Fig. $2 \mathrm{c}$ (grey for uncoupled states, coloured for dressed states); we focus on atoms in the lowest-energy dressed state. Here the atoms' energy (interaction and kinetic) is small compared with the $\approx 10 E_{\mathrm{L}}$ energy difference between the curves; therefore, the atoms remain within the lowest-energy dressed-state manifold ${ }^{5}$, without revealing their spin and momentum components.

In the low-energy limit, $E<E_{\mathrm{L}}$, dressed atoms have a new effective Hamiltonian for motion along $\hat{x}, H_{x}=\left(\hbar k_{x}-q^{*} A_{x}{ }^{*}\right)^{2} / 2 m^{*}$ (motion along $\hat{y}$ and $\hat{z}$ is unaffected); here we choose the gauge where the momentum of the $m_{\mathrm{F}}=0$ component $\hbar k_{x} \equiv p_{\text {can }}$ is the canonical momentum of the dressed state. The red curve in Fig. $2 c$ shows the eigenvalues of $H_{x}$ for $q^{*} A_{x}{ }^{*}>0$, indicating that at equilibrium $p_{\text {can }}=p_{\min }=q^{*} A_{x}{ }^{*}$ (see ref. 2 ). Although this dressed BEC is at rest ( $v=\partial H_{x} / \partial \hbar k_{x}=0$, zero group velocity), it is composed of three bare spin states each with a different momentum, among which the momentum of $\left|m_{\mathrm{F}}=0\right\rangle$ is $\hbar k_{x}=p_{\mathrm{can}}$. None of its three bare spin components has zero momentum, whereas the BEC's momentum - the weighted average of the three-is zero.

We transfer the BEC initially in $\left|m_{\mathrm{F}}=-1\right\rangle$ into the lowestenergy dressed state with $\mathbf{A}^{*}=A^{*} \hat{x}$ (see ref. 2 for a complete technical discussion of loading). At equilibrium, we measure $q^{*} A^{*}=p_{\text {can }}$, equal to the momentum of $\left|m_{\mathrm{F}}=0\right\rangle$, by first removing the coupling fields and trapping potentials and then allowing the atoms to freely expand for a $t=20.1 \mathrm{~ms}$ time of flight (TOF). Because the three components of the dressed state $\left\{\left|k_{x}, m_{\mathrm{F}}=0\right\rangle,\left|k_{x} \mp 2 k_{\mathrm{L}}, m_{\mathrm{F}}= \pm 1\right\rangle\right\}$ differ in momentum by $\pm \hbar 2 k_{\mathrm{L}}$, they quickly separate. Further, a Stern-Gerlach field gradient along $\hat{y}$ separates the spin components. Figure $2 \mathrm{~d}$ shows how the measured and predicted $A^{*}$ depend on the detuning $\delta$. With this calibration, we use $\delta$ to control $A^{*}(t)$.

We realize a synthetic electric field $E^{*}$ by changing the effective vector potential from an initial value $A_{\mathrm{i}}{ }^{*}$ to a final value $A_{\mathrm{f}}{ }^{*}$. We prepare our $\mathrm{BEC}$ at rest with $\mathbf{A}=A_{\mathrm{i}}{ }^{*} \hat{x}$, and make two types of measurement of $E^{*}$. In the first, we remove the trapping potential and then change $A^{*}$ by sweeping the detuning $\delta$ in $0.8 \mathrm{~ms}$, after which the Raman coupling is turned off in $0.2 \mathrm{~ms}$. Thus, $E^{*}$ can 
a
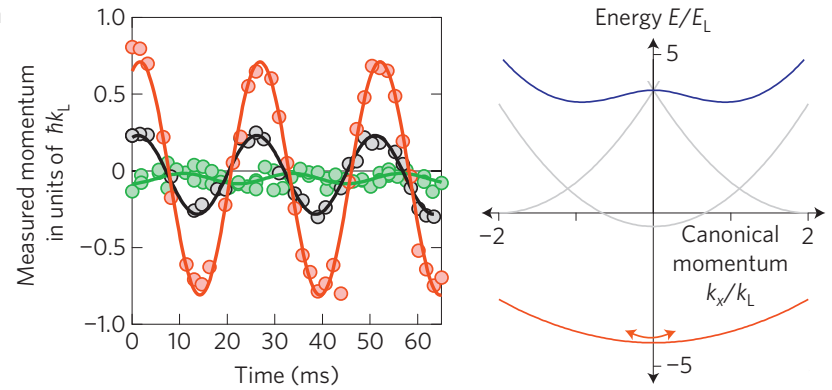

b

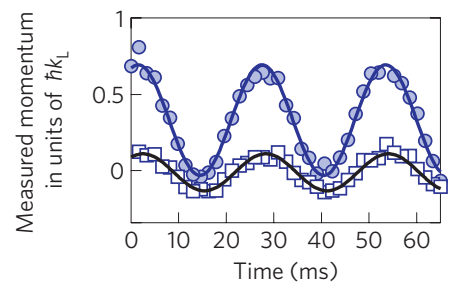

In the second measurement, we examined the time evolution of atoms that remain trapped and strongly dressed after being accelerated by $E^{*}$. We changed $A^{*}$ in $\Delta t \approx 0.3 \mathrm{~ms}$ but left the dressed BEC in the harmonic confining potential for a variable time before the TOF. As the BEC oscillated in the trap, we monitored the out-of-equilibrium canonical momentum $p_{\mathrm{can}}$. It is our access to the internal degrees of freedom-here projectively measuring the composition of the Raman dressed state-that enables the determination of $p_{\text {can }}$. Figure 4 a shows the time evolution of $p_{\text {can }}$ for different $A_{\mathrm{i}}{ }^{*}$ all for $A_{\mathrm{f}}{ }^{*} \approx 0$; as expected, $p_{\text {can }}$ oscillates about $q^{*} A_{\mathrm{f}}{ }^{*}$. As $\Delta t$ is small compared with the $\approx 25 \mathrm{~ms}$ trap period, the change of momentum is dominated by $\Delta p=-q^{*}\left(A_{\mathrm{f}}^{*}-A_{\mathrm{i}}^{*}\right)$, where the contribution from the trapping force is negligible. This translates into an oscillation amplitude $\Delta p$ in both $p_{\mathrm{can}}$ and $m^{*} v=p_{\mathrm{can}}-q^{*} A_{\mathrm{f}}{ }^{*}$ of dressed atoms; the solid crosses in Fig. 3 show the amplitude of the sinusoidal oscillations in $p_{\text {can }}$ versus $A_{\mathrm{f}}{ }^{*}-A_{\mathrm{i}}{ }^{*} \approx-A_{\mathrm{i}}{ }^{*}$, proving that $E^{*}$ has imparted the expected momentum kick.

We repeated the experiment with a non-zero $q^{*} A_{\mathrm{f}}{ }^{*} / \hbar \approx 0.35 k_{\mathrm{L}}$, and observed, as expected, that the oscillations in $p_{\text {can }}$ were offset from zero (Fig. 4b). This illustrates that the observed quantity is not the mechanical momentum $m v$, which should oscillate about zero. We also measured $m v$, where $v$ is the population-weighted average velocity of all spin components (see Methods); although $m v$ does indeed oscillate about zero, the oscillation amplitude is smaller than that of $p_{\text {can }}$. Given the increased effective mass, $m^{*} / m \approx 2.5$, the trap frequency $v_{x}$ along $\hat{x}$ should be reduced by $\sqrt{m / m^{*}}$ from that for undressed atoms, and the oscillation amplitude of $m v$ should be reduced by $m / m^{*}=0.39$ (1) from that of $p_{\mathrm{can}}$. Our results show that $v_{x}^{2}$ is reduced by a factor of $0.38(4)$, as expected, but the momentum oscillation amplitude is reduced by $0.30(2)$, slightly less than predicted (see Methods).

Here we have demonstrated the effects of spatially homogeneous synthetic electric fields; however, this technique is generally applicable to create spatially varying forces. Indeed, as the effective vector potential $A^{*}$ is parameterized by the Raman detuning $\delta$ and coupling $\Omega$, it can be locally patterned through suitable spatially inhomogeneous magnetic bias fields or vector light shifts. Our capability of measuring both the canonical momentum and the mechanical momentum $m v$ is essential. The former characterizes the effective vector potential for dressed spin states, and the latter demonstrates that the dressed atom behaves as a usual particle with an effective mass $m^{*}$ and a well-defined velocity $v$. For atoms initially at rest, as the vector potential is changed with the canonical momentum remaining fixed, the electric field results in a mechanical momentum. For azimuthal vector potentials, such
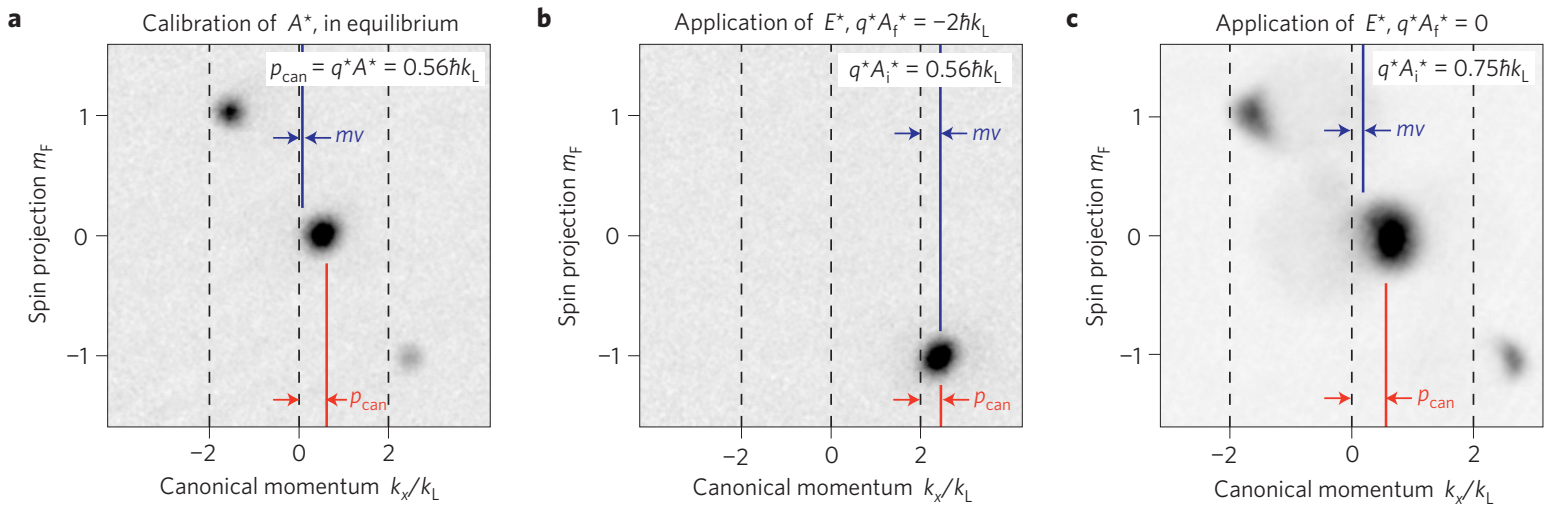

Figure 5 | Example TOF images of the dressed state. $\mathbf{a}, A$ dressed state in equilibrium, where $p_{\text {can }}$ is equal to the vector potential $q^{*} A^{*}$. Here $\hbar \delta=-1.7 E_{\mathrm{L}}$ and correspondingly $q^{*} A^{*}=0.56 \hbar k_{\mathrm{L}}$. Images of this type provide the calibration of $A^{*}$ versus detuning $\delta$ shown in Fig. $2 \mathrm{~d}$. $\mathbf{b}, A$ synthetic electric field $E^{*}$ is applied to the atoms in $\mathbf{a}$, by changing $A^{*}$ from the initial $q^{*} A_{\mathrm{i}}{ }^{*}=0.56 \hbar k_{\mathrm{L}}$ to the final $q^{*} A_{\mathrm{f}}{ }^{*}=-2 \hbar k_{\mathrm{L}}$; the atoms then acquire a momentum $\Delta p \approx 2.56 \hbar k_{\mathrm{L}}$. c, Out-of-equilibrium dressed state where the atoms oscillate in the trap after application of $E^{*}$ by changing $A^{*}$ from $q^{*} A_{\mathrm{i}}{ }^{*}=0.75 \hbar k \mathrm{~L}$ to $q^{*} A_{\mathrm{f}}{ }^{*} \approx 0$. Owing to a larger density of the sample than those in $\mathbf{a}$, scattering halos between $\left|m_{\mathrm{F}}=0\right\rangle$ and $\left|m_{\mathrm{F}}=1\right\rangle$ are visible, indicating interaction during the TOF. 
electric-field-induced mechanical momenta can be used to identify the superfluid fraction of cold-atom systems ${ }^{17}$. In addition, timevarying, alternating vector potentials provide a unique way to drive the trapped BEC. The BEC's response is a measurement analogous to the a.c. transport coefficients of condensed-matter systems.

\section{Methods}

Example TOF images of the dressed state. We measured the momentum of each bare spin component of the dressed state after TOF, during part of which a Stern-Gerlach gradient was applied. Figure $5 \mathrm{a}-\mathrm{c}$ shows example images of the data in Figs 2d, 3 and 4 a, respectively. For the calibration of the vector potential $A^{*}$ from the detuning $\delta$, we use dressed states at equilibrium where $p_{\text {can }}=q^{*} A^{*}$ and measure $p_{\mathrm{can}}$, which is defined as the momentum of $\left|m_{\mathrm{F}}=0\right\rangle$. The mechanical momentum $m v$ is the population-weighted average momentum over all three $m_{\mathrm{F}}$ states, which is nearly zero at equilibrium. This is shown at $\hbar \delta=-1.7 E_{\mathrm{L}}$ and $q^{*} A^{*}=0.56 h k_{\mathrm{L}}$ in Fig. $5 \mathrm{a}$. As $A^{*}$ is changed from the initial value $q^{*} A_{\mathrm{i}}{ }^{*}=0.56 \hbar k_{\mathrm{L}}$ to the final value $q^{*} A_{\mathrm{f}}{ }^{*}=-2 \hbar k_{\mathrm{L}}$, immediately after the trap turnoff, the atoms are accelerated unimpeded by the resulting synthetic electric field $E^{*}$; the final atomic state becomes a nearly pure spin state, $\left|m_{\mathrm{F}}=-1\right\rangle$, with the momentum $\Delta p=m v=-q^{*}\left(A_{\mathrm{f}}{ }^{*}-A_{\mathrm{i}}{ }^{*}\right) \approx 2.56 \hbar k_{\mathrm{L}}$, as illustrated in Fig. $5 \mathrm{~b}$. When the induced $E^{*}$ is applied to trapped atoms, the dressed state is driven out of equilibrium where both $p_{\text {can }}$ and $m v$ oscillate with time (see Fig. $5 \mathrm{c}$ ).

Dynamic change of effective vector potentials. In our first measurement of synthetic electric fields, we observed the momentum imparted by the field kick to the atoms, resulting from a change in the effective vector potential from an arbitrarily chosen $q^{*} A_{\mathrm{i}}{ }^{*}$ to $q^{*} A_{\mathrm{f}}{ }^{*}= \pm 2 \hbar k_{\mathrm{L}}$ (see Fig. 3 ). In principle we could use any $A_{\mathrm{f}}{ }^{*}$ and observe a momentum kick $q^{*}\left(A_{\mathrm{i}}{ }^{*}-A_{\mathrm{f}}{ }^{*}\right)$; however, in general the effective vector potential also depends on the strength of the Raman coupling $\Omega_{\mathrm{R}}$. As a result, extra synthetic electric fields typically appear when $\Omega_{\mathrm{R}}$ is adiabatically turned off. There are three specific cases for which $A^{*}$ does not depend on $\Omega_{\mathrm{R}}$ : when the detuning $\delta=0$ and $\delta \rightarrow \pm \infty$. For the former case, only when $\left|q^{*} A_{\mathrm{i}}{ }^{*}\right|<\hbar k_{\mathrm{L}}$ is there no extra electric force during the removal of $\Omega_{\mathrm{R}}$, where the final atomic state is $\left|m_{\mathrm{F}}=0\right\rangle$. For $\left|q^{*} A_{\mathrm{i}}{ }^{*}\right|>\hbar k_{\mathrm{L}}$, the final atomic state is $\left|m_{\mathrm{F}}= \pm 1\right\rangle$ with an extra momentum of $\mp 2 \hbar k_{\mathrm{L}}$ imparted. Thus in our experiment we changed the vector potential from $q^{*} A_{\mathrm{i}}{ }^{*}$ to $q^{*} A_{\mathrm{f}}{ }^{*}= \pm 2 \hbar k_{\mathrm{L}}$ by changing the detuning from $\delta_{\mathrm{i}}$ to a large $\hbar \delta_{\mathrm{f}}=\mp 60 E_{\mathrm{L}}$; the subsequent turnoff of $\Omega_{\mathrm{R}}$ then exerted no extra forces.

Control of Raman detuning. In all of our experiments, we set the Raman detuning $\delta$ away from resonance through small changes of the bias magnetic field, and hold the $2.32 \mathrm{MHz}$ frequency difference between the Raman beams constant. Because al temporal changes in $\delta$ lead to synthetic electric fields, bias magnetic field noise and relative laser frequency noise can lead to motion in the trap or heating. We phase locked the two Raman beams and observed no change in the heating, showing that relative laser frequency noise is not important in our experiment. However, it is very sensitive to ambient magnetic-field noise, here tied to the $60 \mathrm{~Hz}$ line. This noise gives rise to intractable dynamics of the canonical momentum of the dressed state, where $\delta$ is held constant after the loading. We measured the field noise from the state decomposition of a radiofrequency-dressed state (no Raman fields) nominally on resonance and then feed-forward cancelled the field noise. This reduced the $\sim 0.2 \mu \mathrm{T}$ root-mean-square magnetic-field noise at $60 \mathrm{~Hz}$ by about a factor of 20 , and the remaining root-mean-square field noise is $\sim 0.03 \mu \mathrm{T}$ (including all frequency components up to $\approx 5 \mathrm{kHz}$ ). All of our measurements were made by locking to the $60 \mathrm{~Hz}$ line before loading into the dressed state.

Momentum measurements of the dressed state. The Raman dressed state is a superposition of spin and momentum components; its canonical momentum $p_{\text {can }}$ is the momentum of $\left|m_{\mathrm{F}}=0\right\rangle$. Experimentally, we fit the $m_{\mathrm{F}}=0$ density distribution after the TOF to a Thomas-Fermi profile ${ }^{18}$ and identify $p_{\text {can }}$ as the centre of the distribution. The mechanical momentum of the dressed state $m v$ was measured by a population-weighted average over all three spin states including every pixel with discernible atoms in the image. This takes into account the modification of the TOF density distribution for all $m_{\mathrm{F}}$ states due to interactions during the TOF. Although interactions can exchange momentum between spin states, the total momentum is conserved. Our imaging sensitivity to the $m_{\mathrm{F}}= \pm 1$ atoms is the same to within $5 \%$, which is insufficient to explain the $0.30(2)$ reduction factor in the oscillation amplitude of the mechanical momentum, smaller than the predicted value, $m / m^{*}=0.39(1)$

Received 9 September 2010; accepted 15 February 2011; published online 20 March 2011

\section{References}

1. Jackson, J. D. Classical Electrodynamics (Wiley, 1998).

2. Lin, Y-J. et al. Bose-Einstein condensate in a uniform light-induced vector potential. Phys. Rev. Lett. 102, 130401 (2009).

3. Levin, M. \& Wen, X-G. Colloquium: Photons and electrons as emergent phenomena. Rev. Mod. Phys. 77, 871-879 (2005).

4. Hu, B. L. Emergent/quantum gravity: Macro/micro structures of spacetime. J. Phys.: Conf. Ser. 174, 012015 (2009).

5. Spielman, I. B. Raman processes and effective gauge potentials. Phys. Rev. A 79, 063613 (2009).

6. Juzeliūnas, G., Ruseckas, J., Öhberg, P. \& Fleischhauer, M. Light-induced effective magnetic fields for ultracold atoms in planar geometries. Phys. Rev. A 73, 025602 (2006)

7. Günter, K. J., Cheneau, M., Yefsah, T., Rath, S. P. \& Dalibard, J. Practical scheme for a light-induced gauge field in an atomic Bose gas. Phys. Rev. A 79, 011604 (2009).

8. Cheneau, M. et al. Geometric potentials in quantum optics: A semi-classical interpretation. Europhys. Lett. 83, 60001 (2008).

9. Gerbier, F. \& Dalibard, J. Gauge fields for ultracold atoms in optical superlattices. New J. Phys. 12, 033007 (2010).

10. Juzeliunas, G., Ruseckas, J. \& Dalibard, J. Generalized Rashba-Dresselhaus spin-orbit coupling for cold atoms. Phys. Rev. A 81, 053403 (2010).

11. Ruseckas, J., Juzeliunas, G., Ohberg, P. \& Fleischhauer, M. Non-abelian gauge potentials for ultracold atoms with degenerate dark states. Phys. Rev. Lett. 95, 010404 (2005).

12. Lin, Y-J., Compton, R. L., Jimenez-Garcia, K., Porto, J. V. \& Spielman, I. B. Synthetic magnetic fields for ultracold neutral atoms. Nature 462, 628-632 (2009).

13. Cooper, N. R. Rapidly rotating atomic gases. Adv. Phys. 57, 539-616 (2008).

14. Bloch, I., Dalibard, J. \& Zwerger, W. Many-body physics with ultracold gases. Rev. Mod. Phys. 80, 885-964 (2008).

15. Lin, Y-J., Perry, A. R., Compton, R. L., Spielman, I. B. \& Porto, J. V. Rapid production of ${ }^{87} \mathrm{Rb}$ Bose-Einstein condensates in a combined magnetic and optical potential. Phys. Rev. A 79, 063631 (2009).

16. Hung, C-L., Zhang, X., Gemelke, N. \& Chin, C. Accelerating evaporative cooling of atoms into Bose-Einstein condensation in optical traps. Phys. Rev. A 78, 011604 (R) (2008).

17. Cooper, N. R. \& Hadzibabic, Z. Measuring the superfluid fraction of an ultracold atomic gas. Phys. Rev. Lett. 104, 030401 (2010).

18. Castin, Y. \& Dum, R. Bose-Einstein condensates in time dependent traps. Phys. Rev. Lett. 77, 5315-5319 (1996).

\section{Acknowledgements}

This work was partially supported by ONR, ARO with funds from the DARPA OLE program, and the NSF through the JQI Physics Frontier Center. R.L.C. acknowledges the NIST/NRC postdoctoral program and K.J-G. thanks CONACYT.

\section{Author contributions}

All authors contributed to writing of the manuscript. Y-J.L. led the data-taking effort, in which R.L.C. and K.J-G. participated. W.D.P. and I.B.S. conceived the experiment; I.B.S. supervised this work with consultations from J.V.P.

\section{Additional information}

The authors declare no competing financial interests. Reprints and permissions information is available online at http://npg.nature.com/reprintsandpermissions. Correspondence and requests for materials should be addressed to I.B.S. 